\title{
Avaliação das alterações precoces na coróide e esclera ocorridas em coelhos hipercolesterolêmicos. Estudo histológico e histomorfométrico
}

\author{
Evaluation of choroid and sclera early alterations in hypercholesterolemic \\ rabbits. Histologic and histomorphometric study
}

\author{
Rogil José de Almeida Torres ${ }^{1}$ \\ Maurício Maia ${ }^{2}$ \\ Lucia Noronha ${ }^{3}$ \\ Michel Eid Farah ${ }^{4}$ \\ Andréa Luchini ${ }^{5}$ \\ Décio Brik ${ }^{6}$ \\ Cristina Mucciolir \\ Dalton Bertolin Précoma ${ }^{8}$
}

\begin{tabular}{l} 
RESUMO \\
\hline Objetivo: Demonstrar experimentalmente, através de exames histoló- \\
gicos e histomorfométricos, as alterações degenerativas da esclera e \\
coróide desencadeadas precocemente pela dieta hipercolesterolêmica. \\
Métodos: Coelhos New Zealand foram organizados em dois grupos: GC \\
(grupo controle), composto por 6 coelhos (6 olhos), recebeu dieta \\
normal por 6 semanas; G1, composto por 12 coelhos (12 olhos), tratado \\
previamente com ração colesterol a $1 \%$ (Sigma-Aldrich) por 2 semanas \\
e a partir do 14ㅇ dia com ração colesterol a $0,5 \%$ (Sigma-Aldrich). Os \\
olhos foram submetidos à análise histológica, avaliados com corante de \\
hematoxilina-eosina e ao exame morfométrico. A análise histomor- \\
fométrica foi realizada no setor posterior, adjacente ao disco óptico, e \\
na periferia. Resultados: O GC apresentou espessura média da esclera \\
e coróide na periferia de $228,61 \pm 31,71$ micrômetros, enquanto na \\
região posterior de aproximadamente $246,07 \pm 25,66$ micrômetros. No \\
G1, observou-se espessura média da esclera e coróide na periferia de \\
aproximadamente $303,56 \pm 44,21$ micrômetros, enquanto na região \\
posterior de aproximadamente $295,59 \pm 62,59$. Houve diferença esta- \\
tisticamente significativa da espessura da esclera e coróide entre os \\
grupos na região periférica (p<0,001), não ocorrendo o mesmo no setor \\
posterior(p=0,250). O aumento da espessura da parede de G1 em relação \\
ao GC deve-se principalmente à quantidade elevada de histiócitos e \\
fibras colágenas. Conclusão: Este estudo demonstrou que a dieta \\
hipercolesterolêmica em coelhos induz rapidamente aumento da espes- \\
sura da coróide e esclera, principalmente à custa de histiócitos e fibras \\
colágenas.
\end{tabular}
pina Grande do Sul (PR), no Departamento de Oftalmologia da Universidade Federal de São Paulo e na Pontifícia Universidade Católica do Paraná - PUCPR Curitiba (PR) - Brasil.

${ }^{1}$ Pós-graduando nível Doutorado do Departamento de Oftalmologia da Universidade Federal de São Paulo UNIFESP (SP) - Brasil.

${ }^{2}$ Orientador da pós-graduação do Departamento de Oftalmologia da UNIFESP - São Paulo (SP) - Brasil. Médico Oftalmologista Diretor do Serviço de Cirurgia Vitreorretiniana do Hospital de Olhos Oeste Paulista Assis (SP) - Brasil.

${ }^{3}$ Professora adjunta do Departamento de Patologia da Pontifícia Universidade Católica do Paraná - PUCPR - Curitiba (PR) - Brasil.

${ }^{4}$ Professor adjunto e livre-docente do Departamento de Oftalmologia UNIFESP - São Paulo (SP) - Brasil

${ }^{5}$ Médica do Centro Oftalmológico de Curitiba (PR) Brasil.

${ }^{6}$ Mestre em Oftalmologia pela UNIFESP - São Paulo (SP) - Brasil. Preceptor e responsável pela Residência Médica em Oftalmologia do Hospital Angelina Caron - Curitiba (PR) - Brasil.

${ }^{7}$ Professora livre-docente adjunta do Departamento de Oftalmologia da UNIFESP - São Paulo (SP), Brasil.

${ }^{8}$ Professor adjunto do Departamento de Cardiologia da Pontifícia Universidade Católica do Paraná - PUCPR - Curitiba (PR) - Brasil.

Endereço para correspondência: Rogil José de Almeida Torres. Praça Ruy Barbosa, 827 - Conj 305 - Curitiba (PR) CEP 80010-030

E-mail: rjat@terra.com.br

Fontes de auxílio à pesquisa: Lilacs, Medline e Biblioteca Cocharane. Sem restrição de ano ou linguagem.

Trabalho parcialmente auxiliado pela FAPESP (Fundação de Amparo a Pesquisa do Estado de São Paulo) e pela empresa Kemin do Brasil.

Recebido para publicação em 03.03.2008

Última versão recebida em 17.12.2008

Aprovação em 20.12.2008
Descritores: Colesterol/sangue; Coróide/irrigação sanguínea; Dieta; Hipercolesteremia; Degeneração macular; Aterosclerose; Esclera; Modelos animais; Coelhos; Estudo comparativo

\section{INTRODUCÃO}

A patogênese da degeneração macular relacionada com a idade (DMRI) é controversa e muitas diferentes teorias têm sido propostas ${ }^{(1)}$. Estudos epidemiológicos têm focado a hipótese vascular ao analisar os fatores clássicos de risco para a doença cardiovascular, tais como pressão arterial, colesterol sérico, tabagismo e consequentemente processo aterosclerótico como infarto do miocárdio. Os resultados têm sido incon- 
clusivos, exceto no quesito tabagismo ${ }^{(2)}$. Recente estudo demonstrou que níveis altos de HDL preveniriam a DMRI tardia enquanto alto nível de colesterol total proporcionaria risco maior para DMRI tardia e atrofia geográfica ${ }^{(3)}$. Em modelo animal tem sido demonstrado que a dieta rica em colesterol ocasiona alterações coriorretinianas similares às ocorridas na DMRI humana ${ }^{(4-8)}$. O objetivo deste trabalho é mostrar experimentalmente, através de exames histológicos e histomorfométricos, que as alterações degenerativas da esclera e coróide ocorrem precocemente ao administrar dieta hipercolesterolêmica a coelhos.

\section{MÉTODOS}

Para a realização deste estudo, o protocolo foi aprovado pela Comissão de Ética em Experimentação Animal do Hospital Angelina Caron e Universidade Federal de São Paulo (Protocolo $\mathrm{n}^{\circ}$ 0806/07).

\section{Ambiente de experimentação}

Os procedimentos deste estudo foram realizados nas dependências do laboratório de Técnica Operatória da PUC-PR e do Centro de Estudos do Hospital Angelina Caron (HAC). Os animais foram mantidos no biotério em macroambiente com ciclos de iluminação $12 / 12$ horas, com trocas de ar e temperatura controlada de 19 a $23^{\circ} \mathrm{C}$ e receberam durante o experimento água e ração específica para a espécie $\left(\mathrm{Nuvital}^{\circledR}{ }^{\circledR}\right.$ ) de forma ad libitum.

\section{Animais utilizados}

Foram utilizados 18 coelhos machos albinos (Oryctolagus cunicullus) da linhagem New Zealand, provenientes do biotério da PUC-PR, com os pesos aproximados de $2.500 \mathrm{~g}$, apresentando idade média de 4 meses. Estes animais tiveram um período de adaptação de 7 até 10 dias antes do início do experimento.

\section{Preparo da ração suplementar}

A ração própria para coelho $\left(\mathrm{Nuvital}^{\circledR}\right)$ foi colocada em um recipiente plano e largo, e distribuída numa camada fina. O colesterol diluído foi regado sob a ração, de forma homogênea.

A ração hipercolesterolêmica a $1 \%$, utilizada nas duas primeiras semanas do experimento, apresentava 200 gramas de colesterol (Sigma-Aldrich à $95 \%{ }^{\circledR}$ ) dissolvido em 800 mililitros de clorofórmio (Biotec ${ }^{\circledR}$ ), distribuídos em 20 quilogramas de ração $\mathrm{Nuvital}^{\circledR}$. A ração hipercolesterolêmica a $0,5 \%$, utilizada nas quatro semanas seguintes, apresentava 100 gramas de colesterol dissolvido em 800 mililitros de clorofórmio, distribuídos em 20 quilogramas de ração Nuvital ${ }^{\circledR}$.

Esta ração foi preparada a cada 14 dias, esperando-se 48 horas para ser administrada aos animais. A quantidade diária ofertada para cada animal foi de 600 gramas ao $\mathrm{dia}^{(9)}$.

\section{Delineamento experimental}

Foram organizados dois grupos de coelhos: $\mathrm{O}$ grupo controle denominado GC foi composto por 6 coelhos ( 6 olhos) enquanto o grupo denominado G1 foi composto por 12 coelhos (12 olhos). O GC recebeu dieta padrão, própria para coelho, durante 6 semanas, enquanto G1 recebeu ração colesterol a $1 \%$ por 2 semanas e posteriormente ração colesterol a $0,5 \%$ por 4 semanas.

Cada coelho foi submetido à dosagem sérica de colesterol total, triglicerídeos, HDL colesterol, glicemia de jejum no início do experimento, no $14^{\circ}$ dia e na eutanásia, $42^{\circ}$ dia. As coletas das amostras sanguíneas foram realizadas através de punção da veia marginal auricular magna sob anestesia geral com injeção intramuscular de ketamina $5 \mathrm{mg} / \mathrm{kg}$ e xylazina $35 \mathrm{mg} / \mathrm{kg}$. As dosagens plasmáticas de glicemia, colesterol total, colesterol HDL e triglicerídeos foram realizados pelo método enzimático colorimétrico automatizado (Architec ${ }^{\circledR}$ - Abbott). Uma soroteca com 500 microlitros de cada animal foi congelada para análises futuras.

Os animais foram sacrificados com injeção endovenosa de $5 \mathrm{ml}$ de pentobarbital e os olhos imediatamente fixados em paraformaldeído a $4 \%$ em $0,1 \mathrm{M}$ fosfato / $\mathrm{pH} 7,4$ por 4 horas para análise histopalógica e morfométrica.

\section{Análise histomorfométrica (quantitativa)}

Os dois olhos de cada animal (total de 36) foram removidos e submetidos à fixação, porém somente um foi escolhido para o estudo. Depois da fixação os espécimes foram avaliados macroscopicamente, sendo feito uma secção coronal ao nível do nervo óptico, dividindo os globos oculares em duas metades iguais (superior e inferior). A metade inferior foi estocada para estudos posteriores. Já a metade superior foi submetida à desidratação, diafanização e impregnação em parafina com histotécnico marca Leica ${ }^{\circledR}$, modelo TP 1020. Para a confecção dos blocos de parafina (total de 36) utilizouse o inclusor Leica ${ }^{\circledR}$, modelo EG1160. Estes blocos foram cortados com micrótomo, marca Leica ${ }^{\circledR}$ modelo RM2145, a 5 $\mu$ para obtenção dos cortes histológicos (total de 36). Estes cortes foram pescados em lâmina de vidro com albumina, corados com hematoxilina-eosina e montados com lamínula de vidro de 24x90 mm Entellan, Merck ${ }^{\circledR}$.

As 36 lâminas coradas em hematoxilina-eosina foram analisadas sob o ponto de vista morfológico qualitativo, sendo que foram selecionados 18 cortes histológicos, um para cada animal do estudo, com qualidade técnica adequada para a realização da análise quantitativa.

Para a análise quantitativa, os cortes corados em hematoxilina-eosina previamente selecionados foram avaliados, e com a ajuda da objetiva de 4x e uma caneta de retroprojeção azul, a porção posterior da hemisecção do globo ocular foi dividida, manualmente, em 10 segmentos iguais (de pars plana a pars plana contralateral). Foi então feita a captura de uma imagem por segmento (total de 10 imagens capturadas em cada um dos 18 olhos analisados) através de microscópio Olympus BX50 acoplado a câmera Sony e software Image Proplus ${ }^{\circledR}$. Em cada imagem capturada foram realizadas 4 medidas morfométricas lineares utilizando-se o software Image Proplus ${ }^{\circledR}$ (40 medidas em cada um dos 18 animais avaliados) para avaliar a 
espessura da coróide e da esclera nos 10 segmentos. Finalmente foram realizadas as médias das 4 medidas de cada um dos 10 segmentos em cada um dos 18 espécimes analisados e posteriormente, para efeitos de análise estatística, os 10 segmentos foram agrupados em 2 regiões: regiões próximas ao disco óptico (segmentos A e B), denominada região posterior e; regiões mais próximas a pars plana (zonas $\mathrm{C}, \mathrm{De} \mathrm{E}$ ), denominada região periférica (Figura 1).

\section{Análise estatística}

Para a comparação dos grupos em relação às variáveis do estudo, foi usado o teste não-paramétrico de Mann-Whitney. Para a comparação das regiões analisadas, dentro de cada grupo, foi usado o teste não paramétrico de Wilcoxon. Valores de $\mathrm{p}<0,05$ indicaram significância estatística.

Quanto ao controle metabólico em cada um dos momentos de avaliação, para a comparação dos grupos em relação às variáveis, foi usado o teste não-paramétrico de Mann-Whitney. Valores de $\mathrm{p}<0,05$ indicaram significância estatística.

\section{RESULTADOS}

\section{Resultados laboratoriais (Tabela 1)}

No momento basal, não havia diferença estatisticamente significativa entre os grupos nos quesitos: peso, glicemia, colesterol total, HDL e triglicerídeos. Observou-se, no curso do experimento, que os coelhos do grupo G1 apresentaram

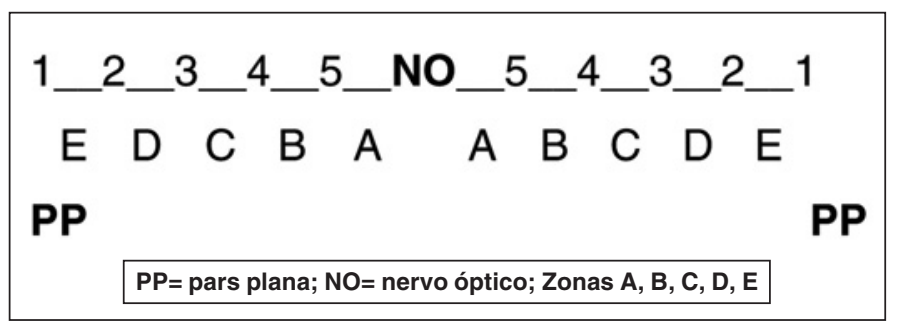

Figura 1 - Esquema dos locais de mensuração morfométrica da esclera e coróide significativo aumento do peso, da glicemia e do colesterol total em relação ao GC.

\section{Microscopia óptica}

Observou-se significativo aumento da espessura do complexo esclera-coróide no G1 (Figura 2B) em relação ao GC (Figura 2A). Com hematoxilina e eosina, foi possível verificar grande quantidade de histiócitos e fibras colágenas na esclera dos coelhos pertencentes ao G1 (Figura 2B).

\section{Grupo controle (GC) X grupo hipercolesterolêmico (G1), para cada região (periférica e posterior)}

Houve significativo aumento da espessura da esclera e coróide do G1. Esta alteração foi mais evidente nos setores periféricos (Tabela 2 e Gráfico 1) que nos setores posteriores (Tabela 2 e Gráfico 2).

\section{Região periférica $X$ região posterior dentro de cada grupo}

A espessura da esclera e coróide posterior do GC foi maior que a espessura deste complexo na região periférica $(\mathrm{p}=0,249)$. No G1 observou-se modificação destes resultados, sendo que a região periférica tornou-se mais espessa que a região posterior ( $\mathrm{p}=0,583)$. Estes dados não adquiriram relevância estatística dentro de cada grupo, exceto no quesito diferença entre a espessura da coróide periférica e posterior no $\mathrm{GC}(\mathrm{p}=0,046)$ (Tabela 3).

\section{DISCUSSÃO}

Acredita-se que a fase inicial da DMRI possa estar relacionada com o acúmulo de depósitos ricos em lipídios abaixo do epitélio pigmentário retiniano (EPR $)^{(1-2)}$. Estes depósitos teriam origem no mau funcionamento das células do $\mathrm{EPR}^{(10)}$ que enviaria restos celulares maiores que o normal, provenientes da fagocitose das extremidades dos fotorreceptores, para a coriocapilar. Estes fragmentos não conseguiriam ultrapassar a membrana de Bruch e se depositariam sobre ela, aumentando sua

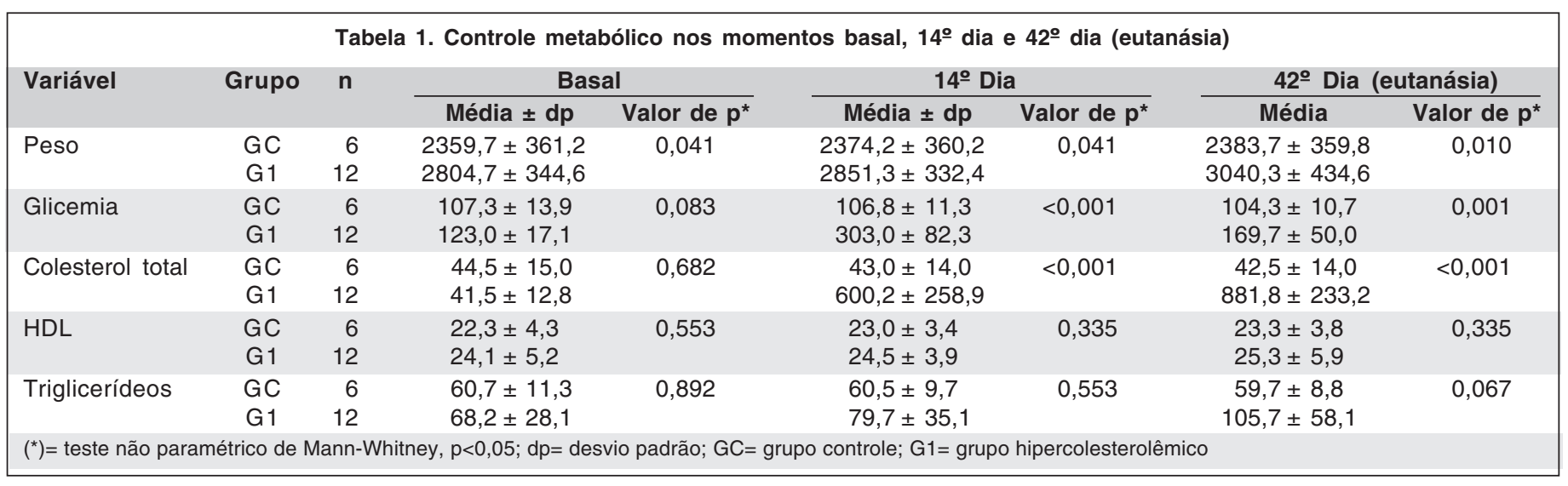




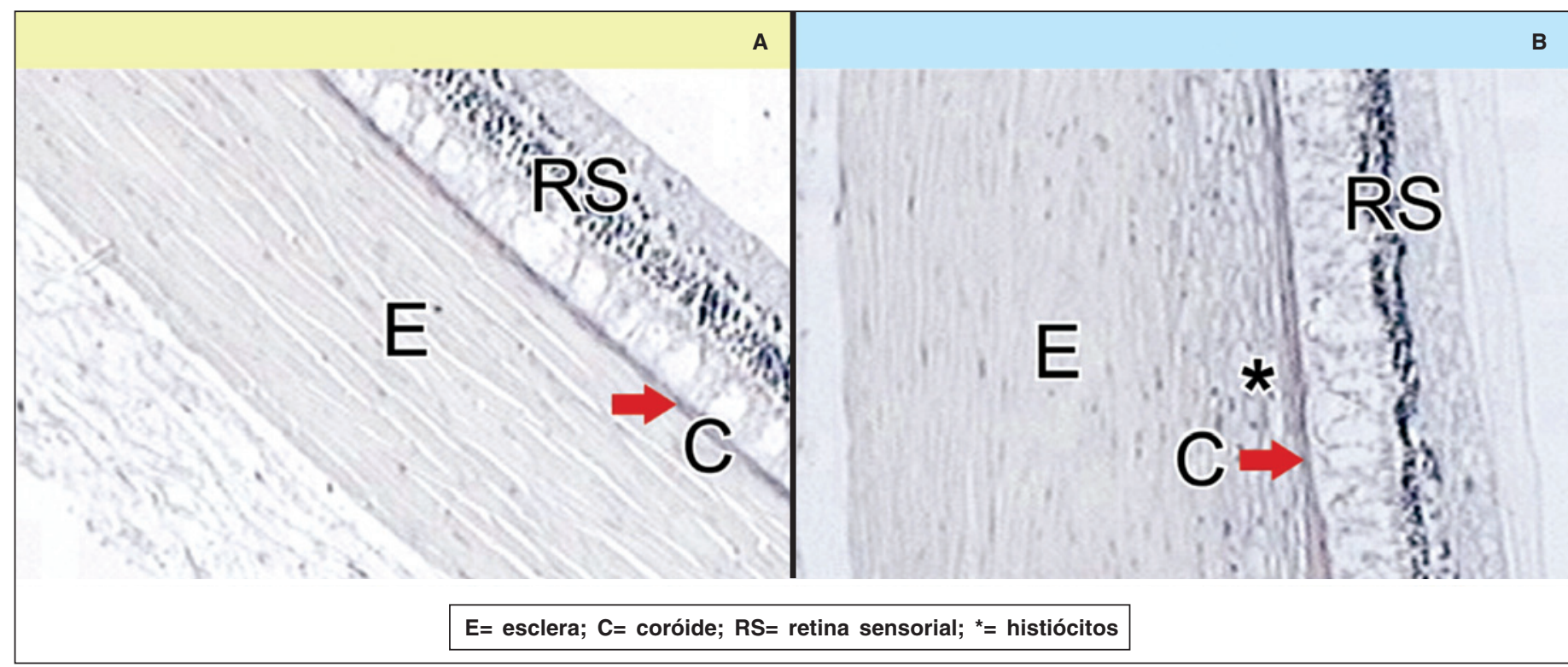

Figura 2 - A) No GC observa-se, com o corante de hematoxilina-eosina, praticamente ausência de histiócitos na esclera. A coróide apresenta sua espessura preservada. B) no G1 observa-se significativo aumento do número de histiócitos e fibras colágenas na esclera. Verifica-se também aumento da espessura da coróide.

Tabela 2. Espessura da esclera e da coróide nas regiões periférica e posterior, em micrômetros

\begin{tabular}{|c|c|c|c|c|c|c|}
\hline Variável & Grupo & $\mathbf{n}$ & \multicolumn{2}{|c|}{ Periférica } & \multicolumn{2}{|c|}{ Posterior } \\
\hline Esclera+coróide & $\begin{array}{l}\mathrm{GC} \\
\mathrm{G} 1\end{array}$ & $\begin{array}{r}6 \\
12\end{array}$ & $\begin{array}{l}228,6 \pm 31,71 \\
303,6 \pm 44,21\end{array}$ & $<0,001$ & $\begin{array}{l}246,1 \pm 25,7 \\
295,6 \pm 62,6\end{array}$ & 0,250 \\
\hline
\end{tabular}

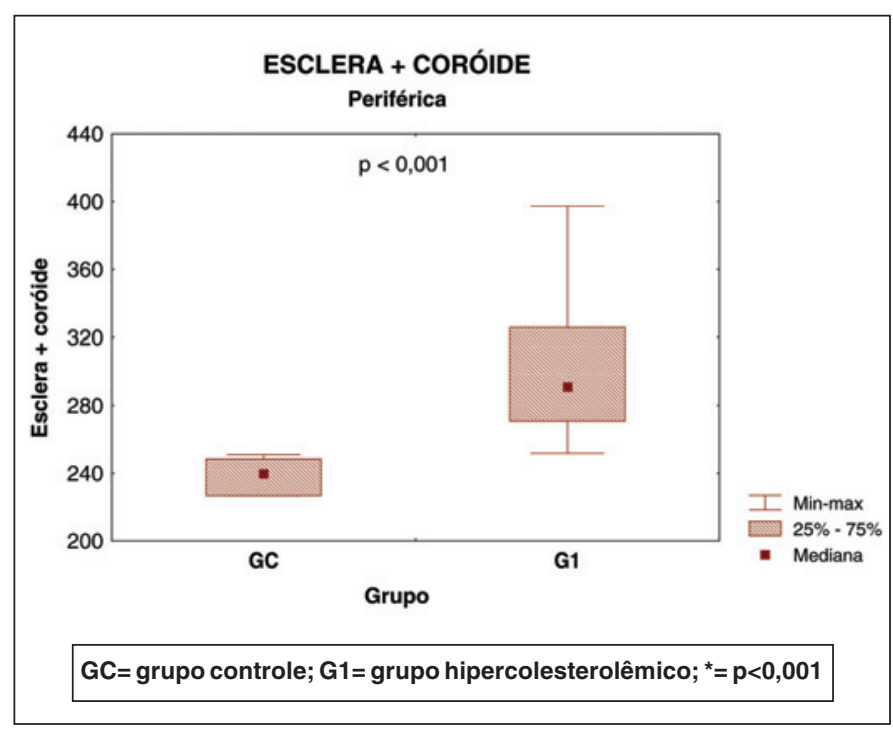

Gráfico 1 - Diferença entre a espessura da esclera e coróide no setor periférico do GC e G1

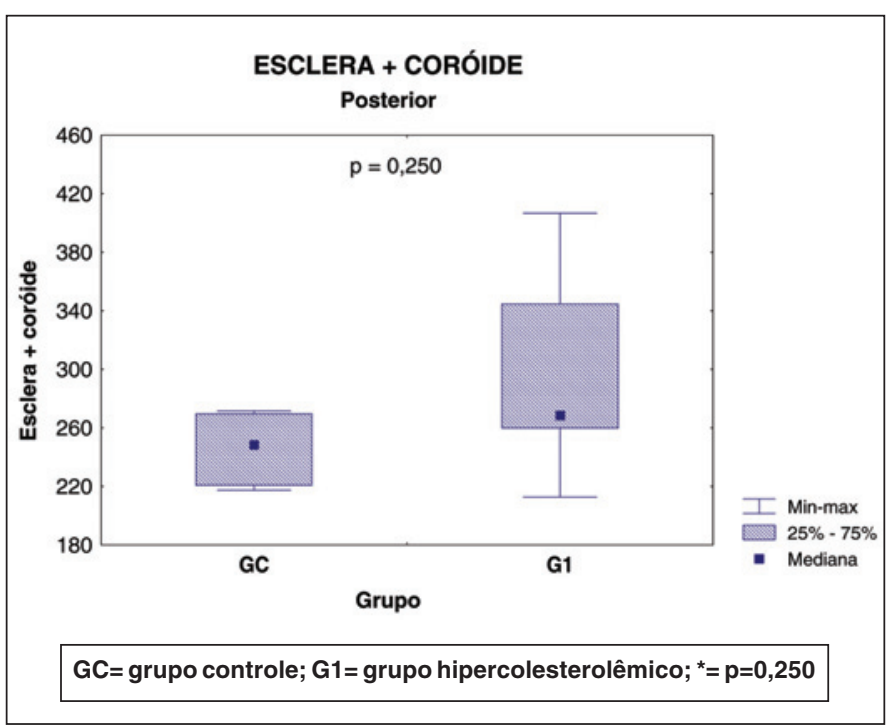

Gráfico 2 - Diferença entre a espessura da esclera e da coróide no setor posterior do GC e G1, em micrômetros 


\begin{tabular}{|c|c|c|}
\hline \multirow[t]{2}{*}{ Variáveis } & \multicolumn{2}{|c|}{$\begin{array}{c}\text { Valor de } p \\
\text { (periférica } x \text { posterior) }\end{array}$} \\
\hline & G C & G1 \\
\hline Esclera periférica $\mathrm{x}$ esclera posterior & 0,249 & 0,530 \\
\hline Coróide periférica $\mathrm{x}$ coróide posterior & 0,046 & 0,209 \\
\hline $\begin{array}{l}\text { Esclera+coróide periférica } \mathrm{x} \\
\text { esclera+coróide posterior }\end{array}$ & 0,249 & 0,583 \\
\hline
\end{tabular}

espessura $^{(11)}$, reduzindo a porosidade e condutibilidade hidráulica $^{(11-13)}$. Isto prejudicaria o acesso de nutrientes da coriocapilar para as células do EPR e fotorreceptores e interferiria na passagem de metabólitos do EPR para a coriocapilar ${ }^{(10)}$.

Entretanto, a teoria proposta neste estudo baseia-se no modelo chamado vascular, similar à fisiopatologia da aterosclerose. Sabe-se que a aterosclerose é um processo reconhecidamente inflamatório, onde o colesterol (LDL) deposita-se na íntima da parede vascular, levando à perda da elasticidade arteriolar. Na esclera, o acúmulo de lipídios ${ }^{(14-15)}$ provoca aumento da resistência pós-capilar da rede vascular da coróide, situada entre a parede escleral progressivamente enrijecida e o conteúdo não compressível do globo ${ }^{(16)}$. A diminuição do fluxo sanguíneo da coróide e o aumento da resistência tendem a elevar a pressão hidrostática da coriocapilar aumentando o vazamento e deposição de proteínas extracelulares e lipídios, particularmente no pólo posterior, formando depósitos basais na membrana de Bruch e drusas; primeiras manifestações clínicas da DMRI ${ }^{(16-17)}$. Na década de 80 , após estudar o coeficiente de rigidez escleral de 29 pacientes com DMRI, esta teoria foi solidificada ${ }^{(18)}$.

A partir destes estudos maior atenção foi dada ao componente escleral na gênese da DMRI. Na década de 90, houve um relato que demonstrou, através de ecodoppler colorido, diminuição da velocidade sanguínea e aumento da pulsação da artéria central da retina e das artérias ciliares posteriores curtas de pacientes que apresentavam $\mathrm{DMRI}^{(16)}$.

Outro estudo, realizado na mesma década, avaliou o fluxo sanguíneo coroideano na região foveolar ${ }^{(19)}$. Foi utilizada a dopplerfluxometria a laser para demonstrar que o fluxo sanguíneo na região foveolar, em pacientes com DMRI, é menor que no grupo controle. Este efeito foi atribuído principalmente à diminuição do volume sanguíneo. Estes estudos demonstraram a diminuição do fluxo sanguíneo da coróide em pacientes com DMRI, sugerindo etiologia isquêmica no desenvolvimento desta doença. É importante ressaltar que existem vários relatos que constatam angiograficamente o comprometimento da perfusão coroideana em pacientes com tal doença ${ }^{(20-24)}$.

É importante lembrar que processos imunológicos e inflamatórios também são observados na DMRI visto que a análise imuno-histoquímica das drusas revela presença de apolipoproteínas B e E, imunoglobulinas, fator X, componente amiloidal P, complemento C5 e C5b-9, fibrinogênio e vitronectin, assim como exames histológicos da membrana neovascular ou cicatriz disciforme mostram a presença de macrófagos adjacentes às áreas adelgaçadas ou roturas da membrana de $\mathrm{Bruch}^{(25-28)}$. Estes fatos podem demonstrar que a aterosclerose e DMRI compartilham de mecanismos fisopatogênicos similares.

Em modelo animal camundongo foi demonstrado que a administração de dieta altamente gordurosa provoca alterações na membrana de Bruch e EPR, comprovando que a ingestão deste tipo de dieta tem potencial para provocar no camundongo alterações similares à DMRI humana ${ }^{(4-5)}$.

A utilização de coelho neste tipo de estudo ganhou preferência em relação a outros animais. Está comprovado que existem semelhanças experimentais entre a aterosclerose neste animal e no ser humano ${ }^{(29)}$. Sabe-se também que os níveis séricos normais de colesterol variam de 25 a $60 \mathrm{mg} \%$ em contraste com o ser humano que apresenta variação de 100 a 200 mg\%. Desta forma o sistema metabólico do coelho pode ser facilmente sobrecarregado através de simples dieta diária hipercolesterolêmica, tornando os experimentos mais viáveis e reprodutíveis $^{(29)}$. Este fato foi constatado no presente relato. O colesterol passou de 41,50 $\mathrm{mg} \%$ para aproximadamente $600 \mathrm{mg} \% \mathrm{em}$ 14 dias e finalmente, no momento da eutanásia, chegou a aproximadamente $881 \mathrm{mg} \%$.

Indícios de alterações esclerais e coroideana provocadas pela hipercolesterolemia em coelhos já foram observados na literatura $^{(29)}$. Um experimento que consistiu na administração diária de $0,75 \%$ colesterol e $2,5 \%$ colesterol a coelhos durante 6 meses demonstrou presença de depósitos lipídicos na superfície interna da coróide assim como células espumosas (histiócitos) e gotículas de lipídios no tecido conectivo. Este estudo ponderou sobre a possibilidade de redução na circulação sanguínea ocular provocada pela hipercolesterolemia ${ }^{(30)}$.

Experimentos posteriores, em olhos de coelhos, surgiram contribuindo para melhor compreensão desta patologia. Um relato $^{(6)}$ demonstrou que a dieta hipercolesterolêmica produz alterações em todas as camadas retinianas, aumento da espessura da membrana de Bruch, corpos densos no citoplasma do EPR, entre outras alterações. Foi concluído que o excesso de colesterol induz a alterações ultraestruturais da retina de coelhos similares àquelas da DMRI humana.

Outro estudo experimental observou que gotículas de colesterol na supracoróide comprimem as camadas vasculares e provocam hipertrofia das células endoteliais e musculares lisas vasculares ${ }^{(8)}$. Neste estudo foi verificado que a espessura supracoroidea está aumentada em coelhos hipercolesterolêmicos devido à abundância de fibras colágenas. No presente relato foi possível evidenciar o significativo aumento da espessura da coróide e da esclera nos coelhos com a dieta hipercolesterolêmica, corroborando a teoria vascular. É importante frisar que os estudos destinados à avaliação das alterações coriorretinianas em olhos de coelhos realizados até a presente data, caracterizaram-se pela administração de dieta colesterol $0,5 \%$ durante o período mínimo de 6 meses $^{(6-8)}$. No presente relato foi possível obter alterações em 6 semanas de dieta hipercolesterolêmica. A dosagem de colesterol administrada neste estudo foi maior que outros relatos nas duas pri- 
meiras semanas (colesterol 1\%), porém iguais aos demais estudos nas últimas 4 semanas do experimento (colesterol $0,5 \%$ ). Este fato ganha importância em nosso meio científico, visto que fica demonstrado que alterações na parede ocular de coelhos podem ser obtidas em curto espaço de tempo, facilitando assim os experimentos e reduzindo os custos das pesquisas.

A espessura do complexo esclera-coróide na região periférica dos coelhos submetidos à dieta hipercolesterolêmica estava significativamente aumentada em relação aos coelhos de dieta normal. A região posterior também demonstrou aumento de espessura no G1, porém sem significância estatística. Estes resultados coincidem com outro relato ${ }^{(8)}$.

Finalmente, vale lembrar que este estudo pode representar a formação da DMRI seca e que experimentos visando à prevenção desta patologia, envolvendo antioxidantes e medicamentos destinados ao controle do colesterol e aterosclerose, poderiam ter seu espaço neste modelo. Desta forma, ressaltase a importância da dieta hipocolesterolêmica na tentativa de se evitar ou atenuar a instalação ou progressão da DMRI.

\section{CONCLUSÃO}

Este estudo demonstrou que a dieta hipercolesterolêmica em coelhos induz rapidamente aumento da espessura da coróide e esclera, principalmente à custa de histiócitos e fibras colágenas.

\section{AGRADEGIMENTOS}

Aos Graduandos da Pontifícia Universidade Católica do Paraná: Luca Rodrigo Pasqualotto, Guilherme Winter, Flávia Kaiber e Greyce Kelly de Souza pela eficiente colaboração prestada em todas as fases do experimento.

À Prof. Dra. Márcia Orlandoswki pela importante colaboração na análise estatística do material coletado.

\section{ABSTRACT}

Purpose: To demonstrate experimentally, by means of histological and histomorphometric examinations, the sclera and choroid degenerative alterations, which take place at an early stage due to a hypercholesterolemic diet. Methods: New Zealand rabbits were divided into two groups: CG (control group) of 6 rabbits ( 6 eyes) received a regular diet for 6 weeks; G1, of 12 rabbits (12 eyes), was first fed a $1 \%$ cholesterol diet (Sigma-Aldrich) for 2 weeks and then from the $14^{\text {th }}$ day on a $0.5 \%$ cholesterol diet (Sigma-Aldrich). The eyes underwent a histological analysis, stained with hematoxiline-eosine, and a morphometric examination. The histomorphometric analysis was performed in the posterior region, adjacent to the optic disk, and in the peripheral region. Results: The CG presented a mean sclera and choroid thickness of $228.61 \pm 31.71$ micrometers in the peripheral region, while the thickness in the posterior region was approximately $246.07 \pm 25.66$ micrometers. In G1, these values were $303.56 \pm 44.21$ micrometers in the peripheral region and $295.59 \pm 62.59$ in the posterior region. There was a statistically significant difference in the sclera and choroid thickness between the groups in the peripheral region $(\mathrm{p}<0.001)$; however, this difference did not occur in the posterior region $(\mathrm{p}=0.250)$. The large number of histiocytes and collagen fibers accounted for the increase of G1 wall thickness in relation to CG. Conclusion: This study demonstrated that the hypercholesterolemic diet in rabbits induces a fast increase in the choroid and sclera thickness, mainly due to the increase in the number of histiocytes and collagen fibers.

Keywords: Cholesterol/blood; Choroid/blood supply; Diet; Hypercholesterolemia; Macular degeneration; Atherosclerosis; Sclera; Models, animal; Rabbits; Comparative study

\section{REFERÊNCIAS}

1. Beatty S, Koh H, Phil M, Henson D, Boulton M. The role of oxidative stress in the pathogenesis of age-related macular degeneration. Surv Ophthalmol. 2000;45(2):115-34.

2. Espinosa-Heidmann DG, Suner IJ, Catanuto P, Hernandez EP, Marin-Castano ME, Cousins SW. Cigarette smoke-related oxidants and the development of sub-RPE deposits in an experimental animal model of dry AMD. Invest Ophthalmol Vis Sci. 2006;47(2):729-37.

3. Tan JS, Mitchell P, Smith W, Wang JJ. Cardiovascular risk factors and the long-term incidence of age-related macular degeneration. Ophthalmology. 2007;114(6):1143-50.

4. Miceli MV, Newsome DA, Tate DJ Jr, Sarphie TG. Pathologic changes in the retinal epithelium and Bruch's membrane of fat-fed atherogenic mice. Curr Eye Res. 2000;20(1):8-16.

5. Ong JM, Zorapapel NC, Rich KA, Wagstaff RE, Lambert RW, Rosenberg $\mathrm{SE}$, et al. Effects of cholesterol and apolipoprotein $\mathrm{E}$ on retinal abnormalities in ApoE-deficient mice. Invest Ophthalmol Vis Sci. 2001;42(8):1891-900.

6. Triviño A, Ramirez AI, Salazar JJ, de Hoz R, Rojas B, Padilla E, et al. A cholesterol-enriched diet induces ultrastructural changes in retinal and macroglial rabbit cells. Exp Eye Res. 2006;83(2):357-66.

7. Ramirez AI, Salazar JJ, De Hoz R, Rojas B, Ruiz E, Tejerina T, et al. Macroglial and retinal changes in hypercholesterolemic rabbits after normalization of cholesterol levels. Expe Eye Res. 2006:83(6):1423-38.

8. Salazar JJ. Ramirez AI, Hoz R, Rojas B, Ruiz E, Tejerina T, et al. Alterations in the choroid in hypercholesterolemic rabbits: reversibility after normalization of cholesterol levels. Exp Eye Res. 2007;84(3):412-22.

9. Sun YP, Lu NC, Parmley WW, Hollenbeck CB. Effects of cholesterol diets on vascular function and atherogenesis in rabbits. Proc Soc Exp Biol Med. 2000;224(3):166-71.

10. Fisher RF. The influence of age on some ocular basement membranes. Eye. 1987;1(Pt 2):184-9.

11. Ramrattan RS, van der Schaft TL, Mooy CM, de Bruijn WC, Mulder PG, de Jong PT. Morphometric analysis of Bruch's membrane, the choriocapillaris, and the choroid in aging. Invest Ophthalmol Vis Sci. 1994;35(6):2857-64.

12. Moore DJ, Hussain AA, Marshall J. Age-related variation in the hydraulic conductivity of Bruch's membrane. Invest Ophthalmol Vis Sci. 1995;36(7): 1290-7.

13. Starita C, Hussain AA, Pagliarini S, Marshall J. Hydrodynamics of ageing Bruch's membrane: implications for macular disease. Exp. Eye Res. 1996; 62(5):565-72.

14. Friedman E. A hemodynamic model of the pathogenesis of age-related macular degeneration. Am J Ophthalmol. 1997;124(5):677-82. Comment in: Am J Ophthalmol. 2000;130(5):658-63.

15. Broekhuyse RM. The lipid composition of aging sclera and cornea. Ophthalmologica. 1975;171(1):82-5. 
16. Friedman E, Krupsky S, Lane A, Oak SS, Friedman ES, Egan K, et al. Ocular blood flow velocity in age-related macular degeneration. Ophthalmology. 1995;102(4):640-6. Comment in: Ophthalmology. 1995;102(8):1125-6.

17. Ambati J, Ambati BK, Yoo SH, Tanchulev S, Adamis AP. Age-related degeneration: etiology, pathogenesis, and therapeutic strategies. Surv Ophthalmol. 2003;48(3):257-93.

18. Friedamn E, Ivry M, Ebert E, Glynn R, Gragoudas E, Seddon J. Increased scleral rigidity and age-related macular degeneration. Ophthalmology. 1989; 96(1):104-8

19. Grunwald JE, Hariprasad SM, DuPont J, Maguire MG, Fine SL, Brucker AJ, et al. Foveolar choroidal blood flow in age-related macular degeneration. Invest Ophthalmol Vis Sci. 1998;39(2):385-90. Comment in: Invest Ophthalmol Vis Sci. 1998;39(11):2201-2.

20. Pauleikhoff D, Chen JC, Chisholm IH, Bird AC. Choroidal perfusion abnormality with age-related Bruch's membrane change. Am J Ophthalmol. 1990; 109(2):211-7.

21. Boker T, Fang T, Steinmetz R. Refractive error and choroidal perfusion characteristics in patients with choroidal neovascularization and age-related macular degeneration. Ger J Ophthalmol. 1993;2(1):10-3.

22. Sarks SH. Aging and degeneration in the macular degeneration: a clinicopathological study. Br J Ophthalmol. 1976;60(5):324-41.
23. Sarks JP, Sarks SH, Killingsworth MC. Evolution of geographic atrophy of the retinal pigment epithelium. Eye. 1988;2(Pt 5):552-77.

24. Chen JE, Fitzke FW, Pauleikhoff D, Bird AC. Functional loss in age-related Bruch's membrane change with choroidal perfusion defect. Invest Ophthalmol Vis Sci. 1992;33(2):334-40.

25. Hageman GS, Luthert PJ, Victor Chong NH, Johnson LV, Anderson DH, Mullins RF. An integrated hypothesis that considers drusen as biomarkers of immune-mediated processes at the RPE-Bruch's membrane interface in aging and age-related macular degeneration. Prog Retin Eye Res. 2001;20(6):705-32.

26. Killingsworth MC, Sarks JP, Sarks SH. Macrophages related to Bruch's membrane in age-related macular degeneration. Eye. 1990;4(Pt 4):613-21.

27. Penfold PL, Killingsworth MC, Sarks SH. Senile macular degeneration: the involvement of immunocompetent cells. Graefes Arch Clin Exp Ophthalmol. 1985;223(2):69-76.

28. van der Schaft TL, Mooy CM, de Bruijn WC, de Jong PT. Early stages of age-related macular degeneration: an immunofluorescence and electron microscopy study. Br J Ophthalmol.1993;77(10):657-61.

29. Cogan DG, Kuwabara T. Ocular changes in the experimental hypercholesterolemia. Arch Ophthalmol. 1959;61:219-25.

30. Janes RG. Changes in the rabbit's eye caused by cholesterol feeding. Am J Ophthalmol. 1964;58:819-28.

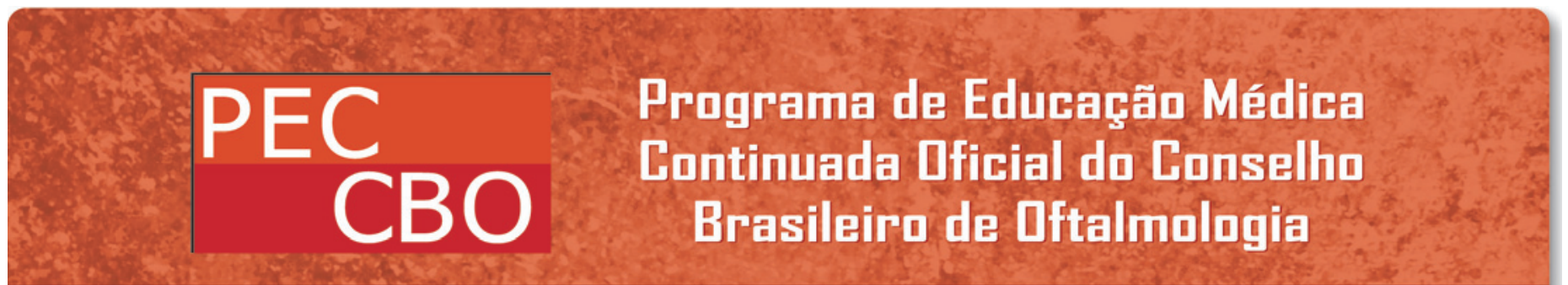

\section{Programa de Edubaf̧án Médica Bontinuada Dficial do Conselho Brasileiru de Dftalmalagia}

